\title{
An Alternative Method for the Measurement of Stellar Nuclear-Reaction Rates
}

\author{
J. O. Fernández Niello ${ }^{1}$, A. Arazi ${ }^{1,2}$, T. Faestermann ${ }^{2}$, K. Knie ${ }^{2}$, G. Korschinek ${ }^{2}$, \\ E. Richter ${ }^{3}$, G. Rugel ${ }^{4}$, and A. Wallner ${ }^{4}$ \\ ${ }^{1}$ Laboratorio TANDAR, C.N.E.A., Av. del Libertador 8250, 1429 Buenos Aires, Argentina \\ ${ }^{2}$ Physikdepartment der Technischen Universität München, 85748 Garching, Germany \\ ${ }^{3}$ Forschungszentrum Rossendorf, Postfach 5101 19, 01314 Dresden, Germany \\ ${ }^{4}$ Ludwig-Maximilians Universität München, Schillerstraße 42, 80336 München, Germany
}

Received on 30 October, 2002

\begin{abstract}
For charged-particle induced reactions occurring in astrophysical scenarios, projectile energies are usually well below the Coulomb barrier of the reacting system. Hence, extremely small cross section reactions pose a difficult task for laboratory measurements. Most commonly, these energy-dependent cross sections are studied by detecting the emitted prompt gamma rays following the de-excitation of the produced compound nucleus. In this work we propose an alternative way for the measurement of the extremely small cross sections of the ${ }^{25} \mathrm{Mg}(\mathrm{p}, \gamma){ }^{26} \mathrm{Al}$ resonant reaction, namely the use of the Accelerator Mass Spectrometry (AMS) technique.
\end{abstract}

\section{Introduction}

In the understanding of the nucleosynthesis of the elements in stars, one of the most important quantities is the reaction rate. This quantity must be evaluated in terms of the stellar temperature $\mathrm{T}$, and its determination involves the knowledge of the excitation function $\sigma(\mathrm{E})$ of the specific nuclear reaction leading to the final nucleus. The particular case of proton capture reactions plays an important role in stellar process of intermediate-mass elements.

In several cases, the formation of a compound nucleus is dominated by a resonant process: the entrance channel, formed by a projectile $\mathrm{P}$ impinging on a target nucleus $\mathrm{A}$, evolves into an excited state of a compound nucleus $\mathrm{B}=\mathrm{P}$ + A, which ultimately decays into lower-lying states with the subsequent emission of gamma rays. This process occurs at well-defined center of mass $(\mathrm{CM})$ energies, $\mathrm{E}^{C M}=$
$\mathrm{E}_{X}-Q$, where $\mathrm{E}_{X}$ is the energy of the excited states of the compound nucleus B and $Q$ is the $Q$-value of the reaction [1].

In a stellar environment, the reaction rate per particle pair is calculated as [2]

$$
<\sigma v>=\int_{0}^{\infty} \sigma v \phi(v) \mathrm{d} v
$$

where $\sigma$ is the energy-dependent reaction cross section, $v$ the relative velocity between the reacting particles and $\phi(v)$ is the velocity distribution of these particles in the stellar plasma.

Nuclei participating in thermonuclear reactions can be considered as non-relativistic and non-degenerate ones, therefore, their velocities $v$ (and hence their energies $E$ ) follow a Maxwell-Boltzmann distribution,

$$
\phi(v)=4 \pi v^{2}\left(\frac{m}{2 \pi k \mathrm{~T}}\right)^{3 / 2} \exp \left(-\frac{m v^{2}}{2 k \mathrm{~T}}\right) \propto \mathrm{E} \exp \left(-\frac{\mathrm{E}}{k \mathrm{~T}}\right)
$$

where $\mathrm{E}=\frac{1}{2} m v^{2}$ represents the kinetic energy of the nucleus with mass $m$, and $\mathrm{T}$ refers to the temperature of the gas. This distribution reaches a maximum value at $\mathrm{E}=k \mathrm{~T}$. The second term defining the reaction rate is the cross section for the compound nucleus formation. This can be described by three factors: a geometrical one, $\sigma=\pi \chi^{2} \propto \mathrm{E}^{-1}$; a sec- ond one resembling the exponential behavior for tunneling through a Coulomb barrier, $\sigma \propto \exp \left(-Z_{P} Z_{A} \mathrm{e}^{2} / \hbar v\right)$; and finally by a nuclear $\mathrm{S}$-factor, $S(\mathrm{E})$, which depicts the specific nuclear characteristics of the particular reaction. Therefore, the reaction rate per particle pair can also be expressed as 


$$
<\sigma v>=\sqrt{\frac{8}{\mu \pi}} \frac{1}{(k \mathrm{~T})^{3 / 2}} \int_{0}^{\infty} S(\mathrm{E}) \exp \left[-\frac{\mathrm{E}}{k \mathrm{~T}}-\frac{\pi \mathrm{e}^{2} Z_{P} Z_{A}}{\hbar} \sqrt{\frac{2 \mu}{\mathrm{E}}}\right] d \mathrm{E}
$$

where $\mu$ is the reduced mass and $Z_{P}$ and $Z_{A}$ are the atomic numbers of projectile and target, respectively.

The exponential term, product of the tunneling probability and the energy distribution of the projectiles, results in the so-called Gamow peak [2], which is centered at

$$
E_{0}=\left[\pi e^{2} Z_{P} Z_{A} \frac{k \mathrm{~T}}{\hbar c} \sqrt{\frac{\mu c^{2}}{2}}\right]^{2 / 3}
$$

and is characterized by a width of

$$
\Delta E_{0}=\sqrt{\frac{16 E_{0} k T}{3}} .
$$

In case of non-resonant reactions (direct capture), the factor $S(E)$ varies slowly with energy and the energy dependence of the reaction rate is therefore characterized only by the Gamow peak. For narrow resonances instead (where, e.g., the total width $\Gamma=\Gamma_{P}+\Gamma_{\gamma}$ is much smaller than the resonant energy $\left.\mathrm{E}_{R}\right)$, which is the case of the ${ }^{25} \mathrm{Mg}(\mathrm{p}, \gamma){ }^{26} \mathrm{Al}$ resonances, the energy dependence of the cross section can be described by the Breit-Wigner expression

$$
\sigma(\mathrm{E})=\frac{\lambda^{2} \omega}{4 \pi} \frac{\Gamma_{P} \Gamma_{\gamma}}{\left(\mathrm{E}-\mathrm{E}_{\mathrm{R}}\right)^{2}+(\Gamma / 2)^{2}},
$$

where $\lambda$ denotes the wavelength of the projectile and $\omega$ is a statistical factor which depends on the spins of projectile $\left(J_{P}\right)$ and target nucleus $\left(J_{A}\right)$ as well as on the angular momentum $J$ of the excited state in the compound nucleus,

$$
\omega=\frac{2 J+1}{\left(2 J_{P}+1\right)\left(2 J_{A}+1\right)} .
$$

Using the Breit-Wigner formula for the cross section, the stellar reaction rate per particle pair yields

$$
<\sigma v>=\left(\frac{2 \pi}{\mu k \mathrm{~T}}\right)^{3 / 2} \hbar^{2} \omega \gamma \exp \left[-\frac{\mathrm{E}_{\mathrm{R}}}{k \mathrm{~T}}\right]
$$

where $\omega \gamma=\omega \Gamma_{P} \Gamma_{\gamma} / \Gamma$ is the resonance strength.

In nuclear astrophysics experiments resonance strengths are usually determined through the on-line detection of the gamma-ray emitted by the reaction products. For the case of a thick target (energy loss much larger than the resonance width) the reaction yield $Y$ is proportional to the resonance strength, which can be obtained as [2]

$$
\omega \gamma=Y \frac{2}{\lambda^{2}} \frac{d \mathrm{E}}{n d x} \frac{M}{M+m}
$$

where $M$ and $m$ are the masses of the target and projectile respectively, $n$ the atomic density and $d \mathrm{E} / d x$ the stopping power.
Due to the barrier penetration factor, the reaction cross section drops exponentially and, hence, the strengths decrease very rapidly with decreasing resonance energy $\mathrm{E}_{R}$. Therefore, an experimental determination of such weak resonances requires high beam intensities and, if gammadetection techniques are applied, an effective suppression of background radiation, too. Effects of cosmic rays within the detector material or cosmic-ray interaction in passiveshielding material around the detectors restrain the lowenergy studies of thermonuclear reactions in the laboratory, since an ubiquitous background level may exceed considerably the reaction-induced counting rate.

In this work we propose to overcome some of such problems arising from the low counting rate, by using the extremely high sensitivity of the accelerator mass spectrometry technique (AMS) for the detection of small amounts of long-lived radioisotopes. In particular, we want to address the stellar occurrence of the ${ }^{25} \mathrm{Mg}(\mathrm{p}, \gamma)$ capture reaction leading to ${ }^{26} \mathrm{Al}$.

\section{The ${ }^{26} \mathrm{Al}$ radioisotope}

The long-lived radioisotope ${ }^{26} \mathrm{Al}^{g}$, with a half-life of $7.2 \times$ $10^{5} \mathrm{yr}$, is a fundamental piece to understand the nucleosynthesis process of intermediate mass nuclei. ${ }^{26} \mathrm{Al}$ is produced both through the beta decay of ${ }^{26} \mathrm{Si}$ and, predominantly, via proton capture on ${ }^{25} \mathrm{Mg}$. In the first case, the ground state of ${ }^{26} \mathrm{Al}\left(J^{\pi}=5^{+}\right)$is bypassed and the decay of ${ }^{26} \mathrm{Si}$ populates an isomeric state ${ }^{26} \mathrm{Al}^{m}\left(T_{1 / 2}=6.35 \mathrm{~s}, J^{\pi}=0^{+}\right)$. Internal transitions from this isomer to the ground state ${ }^{26} \mathrm{Al}^{g}$ are inhibited due to the large spin difference. The only decay mode of this isomer state is the beta decay to the ground state of ${ }^{26} \mathrm{Mg}$, which is free of any $\gamma$-ray emission (see Fig. 1).

Similar spin considerations forbid the decay from the ground state of ${ }^{26} \mathrm{Al}$ to the ground state of the even-even ${ }^{26} \mathrm{Mg}$ nucleus. Beta decay or electron capture from the ground state of ${ }^{26} \mathrm{Al}$ feeds predominantly the first excited state of ${ }^{26} \mathrm{Mg}$. This level decays to the ground state of ${ }^{26} \mathrm{Mg}$ with the emission of a $1.806 \mathrm{MeV}$ gamma ray.

The interest in the study of ${ }^{26} \mathrm{Al}$ nucleosynthesis processes has two milestones: On the one side, the discovery of a ${ }^{26} \mathrm{Mg}$ excess in the Allende meteorite, which was interpreted as an evidence for the live $\beta$-decay of ${ }^{26} \mathrm{Al}$ [3]. The cosmologically short half-life of ${ }^{26} \mathrm{Al}^{g}$ provides a date for the creation of the anomalous material. The other outstanding event was the detection of the $1.806 \mathrm{MeV} \gamma$-ray emission by $\gamma$-ray telescopes and the subsequent mapping of ${ }^{26} \mathrm{Al}$ material within the interstellar medium of our Galaxy [4]. 


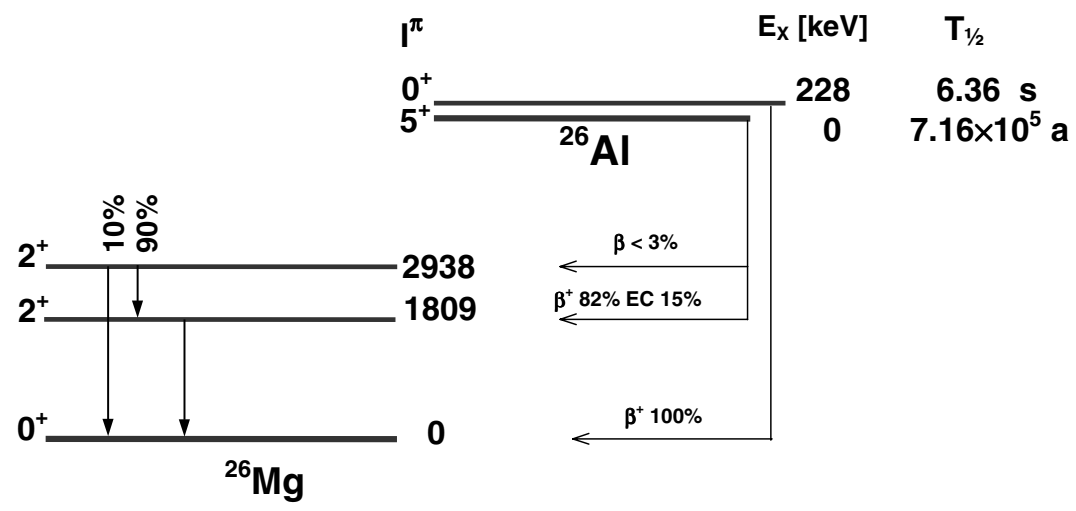

Figure 1. Decay scheme for ${ }^{26} \mathrm{Al}$. The ground state decays predominantly to the first state of ${ }^{26} \mathrm{Mg} J^{\pi}=2^{+}\left(\mathrm{E}_{X}=1809 \mathrm{keV}\right)$. The residual part of the $\beta^{+}$-decay (3\%) feeds the second excited state of ${ }^{26} \mathrm{Mg}$ at an excitation energy of $\mathrm{E}_{X}=2938 \mathrm{keV}\left(J^{\pi}=2^{+}\right)$.

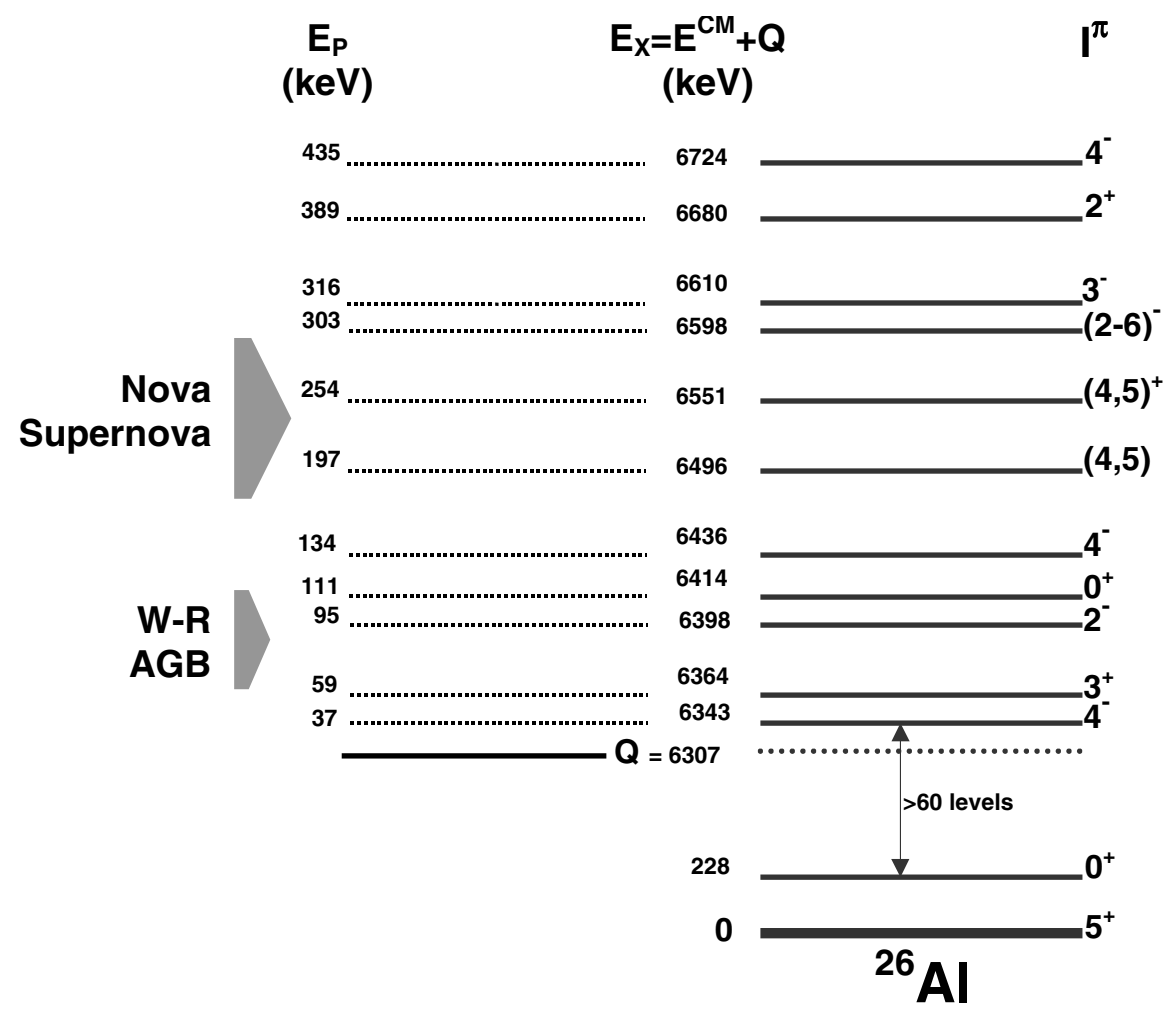

Figure 2. Level scheme of ${ }^{26} \mathrm{Al}$ above the ${ }^{26} \mathrm{Mg}+\mathrm{p}$ threshold. Also shown in this figure are the proton energies (in the laboratory system) that match the resonances. The arrows in the left part indicate the Gamow energy for the case of novae and supernovae and Wolf-Rayet or AGB stars and their widths resemble the width of the Gamow peak (see text).

Astrophysical sources of ${ }^{26} \mathrm{Al}$ production within our Galaxy are not well established. Possible production sites are supernovae, novae or massive stars (in the Asymptotic Giant Branch or in the Wolf Rayet phases). The proton temperature of supernovae or novae is in the order of $\mathrm{T}=$ $2 \times 10^{8} \mathrm{~K}$, whereas in massive stars hydrogen burning occurs at temperatures around $\mathrm{T}=5 \times 10^{7} \mathrm{~K}[5]$. These temperatures correspond to proton energies of around $200 \mathrm{keV}$ and $100 \mathrm{keV}$, respectively (see Eq. 4). Hence, solving the controversy concerning potential production sites of ${ }^{26} \mathrm{Al}$ demands a precise determination of the production rate for the capture reaction ${ }^{25} \mathrm{Mg}(\mathrm{p}, \gamma)$ over the above-mentioned proton energies.
The Coulomb barrier for the $\mathrm{p}+{ }^{25} \mathrm{Mg}$ reaction in the center-of-mass system is $2860 \mathrm{keV}$, i.e. the energies of interest are well below it. Within this range, the ${ }^{25} \mathrm{Mg}(\mathrm{p}$, $\gamma$ ) reaction is dominated by the production through narrow resonances, which correspond to states near the proton threshold in the compound nucleus $(Q=6307 \mathrm{keV})$, see Fig. 2. The ${ }^{25} \mathrm{Mg}(\mathrm{p}, \gamma)^{26} \mathrm{Al}$ reaction was studied at proton energies down to $198 \mathrm{keV}$ by means of the prompt $\gamma$-ray detection technique [7, 8], see Table I. This lower value of the projectile energy matches to a resonant state located at $\mathrm{E}_{x}=6496 \mathrm{keV}$. Resonances below this level (existing at 6436, 6415, 6399, 6364, and $6343 \mathrm{keV}$ ) could not be measured by this technique. Their strengths 
were, instead, estimated from spectroscopic factors provided by the ${ }^{25} \mathrm{Mg}\left({ }^{3} \mathrm{He}, \mathrm{d}\right){ }^{26} \mathrm{Al}$ reaction $[9,10]$. Therefore, ${ }^{25} \mathrm{Mg}(\mathrm{p}, \gamma){ }^{26} \mathrm{Al}$ measurements were done only for resonant states populated in supernova or nova events and no direct information is available for those resonance strengths which determine the hydrostatic hydrogen burning in massive stars.

Table 1. Resonance strengths for the ${ }^{25} \mathrm{Mg}(\mathrm{p}, \gamma){ }^{26} \mathrm{Al}$ reaction. The first column indicates the proton energies in the laboratory system. The second column gives the corresponding excitation energies for the excited states of ${ }^{26} \mathrm{Al}$ above threshold. Column three gives the results for $\omega \gamma$ from previous works [6].

\begin{tabular}{ccr}
$\mathrm{E}_{P}(\mathrm{keV})$ & $\mathrm{E}_{X}(\mathrm{keV})$ & $\omega \gamma(\mathrm{eV})$ \\
\hline 434.6 & 6724 & $0.9 \pm 0.2$ \\
389.0 & 6680 & $0.40 \pm 0.05$ \\
316.1 & 6610 & $0.07 \pm 0.01$ \\
303.5 & 6598 & $(5.1 \pm 0.6) \times 10^{-5}$ \\
254.0 & 6551 & $(5.1 \pm 0.6) \times 10^{-6}$ \\
197.0 & 6496 & $(7.4 \pm 0.9) \times 10^{-7}$ \\
135.1 & 6436 & $\left.\leq 1.5 \times 10^{-10} a\right)$ \\
112.3 & 6414 & $\left.2.1 \times 10^{-11 a} a\right)$ \\
95.8 & 6398 & $1.2 \times 10^{-10 a}$ \\
59.8 & 6364 & $\left.2.8 \times 10^{-13 a} a\right)$ \\
38.4 & 6343 & $2.4 \times 10^{-20 a} a$
\end{tabular}

${ }^{a)}$ Values determined using the ${ }^{25} \mathrm{Mg}\left({ }^{3} \mathrm{He}, \mathrm{d}\right){ }^{26} \mathrm{Al}$ reaction

\section{The proposed method}

AMS is a very sensitive technique capable of tracing longlived radioisotope concentrations (ratio of the unstable to the stable nuclei) down to levels of about $10^{-15}$. This sensitivity is used to perform an off-line measurements of ${ }^{26} \mathrm{Al}$ nuclei formed when ${ }^{25} \mathrm{Mg}$ target foils are bombarded with highintensity low-energy proton beams. The ${ }^{26} \mathrm{Al}$ ions produced by this reaction are stopped and collected in the foil. After the irradiation, these targets are chemically processed in order to be used as sample material in an ion source. In this way, small amounts of produced ${ }^{26} \mathrm{Al}$ nuclei can be detected by means of the AMS technique at the Munich MP tandem accelerator.

It should be noted that in this procedure we measure the production of the ${ }^{26} \mathrm{Al}$ in its ground state, which is the actual source of the $1.806 \mathrm{MeV}$ gamma ray and, therefore, the main issue of astrophysical interest. The produced ${ }^{26} \mathrm{Al} \mathrm{nu-}$ clei in the isomeric state decay to ${ }^{26} \mathrm{Mg}$ before the AMS measurement takes place. In the following we will discuss in more detail the relevant steps in this procedure, such as target construction, proton beam energy, chemical treatment of the Mg-targets and the AMS measurement itself.

The projectile energy is chosen such that all protons meet the resonant energy within the target thickness, taking into account the energy loss and dispersion due to straggling of protons in the target material. The targets themselves are made of ${ }^{25} \mathrm{MgO}$ (enriched to $93 \%$ ), evaporated on a glasscarbon backing. $\mathrm{MgO}$ is chemically more stable than atomic magnesium and it has a lower sputtering rate when bombarded by low-energy protons. Due to the same sputtering considerations a large target area $\left(4 \times 4 \mathrm{~cm}^{2}\right)$ is used.

In the AMS measurement, the ${ }^{26} \mathrm{Al}$ counting rate (typically lower than one atom per minute) must be normalized to the intensity of the ${ }^{27} \mathrm{Al}$ beam. Hence, ${ }^{27} \mathrm{Al}$ carrier material must be added in a well known amount, which can be estimated as follows: Considering a typical integrated proton dose onto the ${ }^{25} \mathrm{MgO}$ target, an amount of about $10^{5}$ atoms of ${ }^{26} \mathrm{Al}$ is produced. In order to be measurable, the ${ }^{26} \mathrm{Al} /{ }^{27} \mathrm{Al}$ ratio has be $\geq 10^{-14}$, well above the natural background level of any $\mathrm{Al}$ material. Hence, the ${ }^{27} \mathrm{Al}$ carrier should be limited to $100 \mu \mathrm{g}$ or even less.

The ${ }^{26} \mathrm{Al}$ radioisotope has a stable isobar, ${ }^{26} \mathrm{Mg}$. Although enriched ${ }^{25} \mathrm{Mg}$ targets are used, macroscopic amounts (about $2 \%$ ) of ${ }^{26} \mathrm{Mg}$ are present. This isobaric interference is partially avoided through a very careful chemical separation technique. The sample material is dissolved in $\mathrm{HCl}$ and three successive precipitations are done adding $\mathrm{NH}_{3}$, each followed by several washings with extremely pure water $(<0.5 \mathrm{ppb} \mathrm{Mg})$. By these means, a reduction of $\mathrm{Mg}$ down to $5 \mathrm{ppm}$ on $500 \mu \mathrm{g}$ samples is achieved.

Most commonly, ${ }^{26} \mathrm{Al}$ AMS-measurements are performed producing negative atomic $\mathrm{Al}$ beams in the ion source because $\mathrm{Mg}$ negative ions are unstable and, therefore, interference from the isobar ${ }^{26} \mathrm{Mg}$ is avoided. However, in this case, the small amount of ${ }^{26} \mathrm{Al}$ atoms to be measured requires an optimization of the ion source performance. Therefore we used $\mathrm{AlO}^{-}$ions, as the yield of negative oxide ions from the ion source turned to be around 25 times larger than that of the atomic ones, $\mathrm{Al}^{-}$. Molecular ions are dissociated during the stripping process at the terminal of the tandem accelerator. In this case, however, ${ }^{26} \mathrm{Mg}$ is injected as well, since $\mathrm{MgO}^{-}$ions are prolific and stable, and ${ }^{26} \mathrm{Al}$ ions need then to be separated from ${ }^{26} \mathrm{Mg}$ effectively at the final stage of the AMS analyzing system[11]. It should be noted that ${ }^{26} \mathrm{Mg}$ ions have the same magnetic rigidity and velocity as their isobar ${ }^{26} \mathrm{Al}$, and hence they circumvent the beam analysis systems (analyzing magnet, Wien filter, etc.) throughout the accelerator beam line.

The mass difference between ${ }^{26} \mathrm{Mg}$ and ${ }^{26} \mathrm{Al}$ $\left(\Delta M / M \approx 1 \times 10^{-4}\right)$ is too small for an efficient separation in the AMS facilities and, therefore, special detection techniques to distinguish these isobars are required. Our measurements are performed at a final particle energy of $95 \mathrm{MeV}$ from the tandem accelerator for the selected ${ }^{26} \mathrm{Al}^{7+}$ ions. This allows isobar separation by means of a gas-filled magnet. Finally the ${ }^{26} \mathrm{Al}$ ions were identified by their position, residual energy, differential energy losses and incident angle with a gas ionization chamber placed at the exit of the magnet[12].

Up to now, resonances corresponding to proton energies of $\mathrm{E}_{P}=434 \mathrm{keV}, 389 \mathrm{keV}$ and $316 \mathrm{keV}$ have been measured[13]. Our results are in good agreement with the values measured by the on-line gamma-detection technique (see fig. 3). Measurements for further resonances, among them one at $96 \mathrm{keV}$, are planned for the near future. 


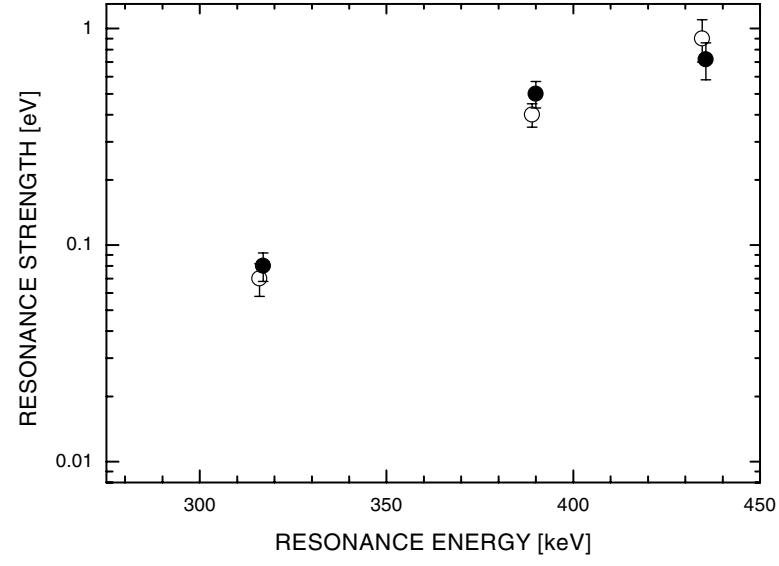

Figure 3. Preliminary AMS results [13](full circles) and the accepted values [6] (open circles) of the resonance strengths $\omega \gamma$ at proton laboratory energies of 316,389 , and $434 \mathrm{keV}$.

\section{Conclusions}

Summarizing, we expect that the ultra high sensitivity of AMS will allow us to overcome some of difficulties arising from the gamma background of on-line determination of extremely low cross sections. This method was proven by measuring resonances at 434, 389 and $316 \mathrm{keV}$ for the ${ }^{25} \mathrm{Mg}(\mathrm{p}, \gamma){ }^{26} \mathrm{Al}$ reaction. However, in order to address the main question of this study, whether the ${ }^{26} \mathrm{Al}$ production stems from nova or from AGB or Wolf-Rayet stars, cross sections for lower proton energies have to be determined experimentally. For this reason, measurements of this reaction cross section down to $96 \mathrm{keV}$ are in progress.

\section{References}

[1] C. E. Rolfs, H. P. Trautvetter, and W. S. Rodney, Rep. Prog. Phys. 50, 233 (1987).

[2] C. E. Rolfs and W. S. Rodney, Cauldrons in the Cosmos (The University of Chicago Press, Chicago, 1988).

[3] G.J. Wasserburg and D.A. Papanastassiou, Essays in Nuclear Astrophysics, ed. C.A. Barnes, D.D. Clayton, and D.N. Schramm, (Cambridge University Press, 1982).

[4] W. Mahoney, J.C. Ling, A.S. Jacobson, and R. Lingenfelter, Ap. J. 262, 742 (1982).

[5] N. Prantzos and R. Diehl, Phys. Rep. 267, 1 (1996).

[6] C. Iliadis, L. Buchmann, P.M. Endt, H. Herndl, and M. Wiescher, Phys. Rev. C53, 475 (1996).

[7] K. Elix, H.W. Becker, L. Buchmann, J. Görres, K.U. Kettner, M. Wiescher, and C. Rolfs, Z. Phys. A293, 261 (1979).

[8] C. Iliadis, Th. Schange, C. Rolfs, U. Schröder, E. Somorjai, H.P. Trautvetter, K. Wolke, P.M. Endt, S.W. Kikstra, A.E. Champagne, M. Arnould, and G. Paulus, Nucl. Phys. A512, 509 (1990)

[9] A.A. Rollefson, V. Wijekumar, C.P. Browne, M. Wiescher, H.J. Hausman, W.Y. Kim, and P. Schmalbrock, Nucl. Phys A507, 413 (1990).

[10] A.E. Champagne, A.J. Howard, M.S. Smith, P.V. Magnus, and P.D. Parker, Nucl. Phys. A505, 384 (1989).

[11] A. Arazi, T. Faestermann, J. Fernández Niello, K. Knie, G. Korschinek, E. Richter, G. Rugel, and A. Wallner, New Astronomy Rev. 46, 525 (2002).

[12] K. Knie, T. Faestermann, G. Korschinek, G. Rugel, W. Rühm, and C. Wallner, Nucl. Instr. and Meth. B172, 717 (2000).

[13] A. Arazi, T. Faestermann, J. O. Fernández Niello, K. Knie, G. Korschinek, E. Richter, G. Rugel, and A. Wallner, to be published. 